This is an Open Access article distributed under the terms of the Creative Commons Attribution-Noncommercial License, which permits unrestricted use, distribution, and reproduction in any noncommercial medium, provided the original work is properly cited.

\title{
THE EFFECTIVENESS OF MID/FAR IR BLIND, WIDE AREA, SPECTRAL SURVEYS IN BREAKING THE CONFUSION LIMIT
}

\author{
G. Raymond ${ }^{1}$, K. G. Isaak ${ }^{1}$, D. Clements ${ }^{2}$, A. Rykala ${ }^{1}$, and C. Pearson ${ }^{3,4}$ \\ ${ }^{1}$ Cardiff University, School of Physics \& Astronomy, Queens Buildings, The Parade, Cardiff, CF24 3AA, UK \\ ${ }^{2}$ Physics Department, Imperial College London, Prince Consort Road, London SW7 2AZ, UK \\ ${ }^{3}$ Space Science and Technology Department, CCLRC Rutherford Appleton Laboratory, Didcot, Oxfordshire, OX11 0QX, UK \\ ${ }^{4}$ Department of Physics, University of Lethbridge, 4401 University Drive, Lethbridge, Alberta, T1J 1B1, Canada
}

\section{ABSTRACT}

Source confusion determines the useful depth to which to take large-area extragalactic surveys. 3D imaging spectrometers, with positional as well as spectral information, however, can potentially provide a means by which to break the traditional confusion limit.

In this poster we present the results of our investigation into the effectiveness of mid/far infrared, blind, wide area spectroscopic surveys with SAFARI in breaking through the confusion limit. We generate an artificial sky representative of 100 SAFARI footprints based on two galaxy evolution models: bright-end and burst-mode. Using a fully automated redshift determination method we find we can accurately estimate redshifts for 37 and $53 \%$ of sources with continuum fluxes that are as much as an order of magnitude below the traditional continuum confusion limit (at $120 \mu \mathrm{m}$ ) for the bright-end and burst-mode evolution models respectively. Our results suggest that deep, blind spectral line surveys with SAFARI will be able to break the traditional photometric confusion limit, allowing us to resolve a significant number of previously inaccessible galaxies, and so potentially to differentiate between different galaxy evolution models.

Key words: Galaxies: formation - Missions: SPICA

\section{INTRODUCTION}

Source confusion may be defined as the degradation of the quality of photometry of sources projected on a scale to the order of the telescope beam size (Scheuer, 1957). The confusion limit sets the useful depth to which large-area extra-galactic surveys should be taken. For example, the Herschel mission (Pilbratt, 2004) (launched in 2009) has a $3.5 \mathrm{~m}$ diameter mirror which realizes an angular resolution of $\sim 8$ " at $120 \mu \mathrm{m}$. At these wavelengths, the confusion limit for such a mirror is estimated to be around $\sim 5 \mathrm{mJy}$ (Dole et al., 2004; Jeong et al., 2006; Le Borgne et al., 2009). Surveys at $24 \mu \mathrm{m}$ suggest that at these flux levels one will only be able to resolve at most $\sim 50 \%$ of the cosmic infrared background (CIB) (Dole et al., 2004; Le Borgne et al., 2009). It is possible to reduce the confusion limit at a given wavelength by by increasing the diameter of the mirror. However, far-infrared (FIR) observations are necessarily made from space, which places a practical limit on the telescope diameter and thus by how much we can reduce the confusion limit.

One way to break the confusion limit makes use of the extra dimension of wavelength, to which one has access in spectroscopic surveys. Discrete sources can be identified by relatively bright, narrow-band emission lines: by comparing rest-wavelengths with observed wavelengths one can uniquely determine redshift.

A preliminary study to explore the efficacy of blind, wide area, spectroscopic surveys in identifying FIR sources is described in Clements et al. (2007). In this work an artificial 'sky' was populated with spectra based on template FIR/mid-infrared (MIR) spectral energy distributions (SEDs) with FIR emission line strengths taken from ISO-LWS observations. The 'sky' was observed using instrumental parameters matched with those of SAFARI (i.e. field of view, spectral resolution, sensitivity, etc.). Redshifts were determined by hand by comparing the positions of the $[\mathrm{OI}]$ and $[\mathrm{CII}]$ lines, at $63.2 \mu \mathrm{m}$ and 157.7 $\mu \mathrm{m}$ respectively, to the position of the peak of the SED. Clements et al. found that it was possible to accurately estimate redshifts for sources as much as $\sim 5$ times below the traditional continuum confusion limit at $120 \mu \mathrm{m}$. Sources at redshifts $z<2.5$ and with $120 \mu \mathrm{m}$ flux $S_{120 \mu m}>1 \mathrm{mJy}$ were accurately estimated with $100 \%$ efficiency.

In this work we examine a new, much larger artificial 'sky' populated with better template SEDs with both FIR/MIR emission lines and employ a new automated method of evaluating source redshifts in a time efficient manner. By way of comparison a Figure of $\sigma_{c}(\lambda=120$ $\mu \mathrm{m})=4.3 \mathrm{mJy}$ is adopted for the confusion limit, based on Dole et al. (2004).

\section{Method}

\subsection{Generation of Artificial Skies}

We generate 100 artificial 2'x2' skies (see Swinyard these proceedings) commensurate with the instrumental parameters of SAFARI, for two different galaxy evolution models: the bright-end and burst mode evolution models of Pearson (2005) and Pearson et al. (2007). These evolution models broadly divide galaxies into 4 spectral categories: those of normal, starforming, luminous / ultraluminous infrared galaxies (LIRGS/ULRGS) and active 
galactic nuclei (AGN). These spectral categories are also further subdivided into 11 different classes of object: normal cold, normal, starburst M82, starburst, $10^{11} L_{\odot}$ LIRG, $10^{11.5} L_{\odot}$ LIRG, cold LIRG, hot ULIRG, cold ULIRG, Seyfert 1 and Seyfert 2. The bright-end model assumes evolution in the galaxy population in both the density and luminosity for sources modeled by a simple power law. The evolutionary model is an updated framework of that first presented in Pearson \& Rowan-Robinson (1996), with star-forming galaxies rather than ULIRGs (ultra luminous infrared galaxies) dominating in $15 \mu \mathrm{m}$ selected source counts. The burst mode evolutionary model predicts that the upturn of emission at $15 \mu \mathrm{m}$ (Elbaz et al., 1999) and peak at at $24 \mu \mathrm{m}$ (Papovich et al., 2004) is to due the emergence of a new population of U/LIRGs. The burst mode evolutionary model presented by Pearson (2001) has power law evolution similar to the brightend model for the starburst and AGN sources and an initial violent exponential evolutionary phase from $z=0$ to $z=1$ followed by a power law evolutionary phase for the UILRGs. Both the bright-end and burst mode evolutionary models have non-evolving normal galaxy populations. The spectral type vs flux/redshift distributions are shown in Figure 1.

These skies are populated with the same SEDs as used by Clements et al. MIR emission lines are taken from the same sources as used to determine FIR emission lines, except in a few cases where data was not available at MIR wavelengths, in such cases MIR spectroscopic observations of sources with similar FIR characteristics were used. Instrumental noise at a level commensurate with 10 hours of SAFARI integration time $(1 \sigma=0.342 \mathrm{mJy}, \mathrm{R} \sim 1000$ at $120 \mu \mathrm{m})$ is then added. In order to take into account the variance in results due to different noise, 10 differently seeded randomly generated Gaussian noise arrays are generated for each spectrum. We therefore have a total of 1000 different datacubes for each evolutionary model. Simultaneously a 'truth' catalog is generated for each datacube/FoV, in order to compare the redshifts we estimate to their input values.

The artificial skies also include spatial bins where more than one source is present. If the fluxes of the two sources are comparable then their spectra lines become effectively mixed, making it difficult to determine which emission lines come from which objects. The work described in Clements et al. (2007) did not address this problem. In this work we try to extract redshifts from both spectrally, and spatially confused sources. Throughout this work we refer to the brightest source in a spatial bin as the primary, and the second brightest as the secondary.

\subsection{Extraction OF Redshifts}

In the 2000 realizations of the SAFARI footprints there are $\sim 15000$ sources with $S_{120 \mu m}>0.342 \mathrm{mJy}$. We therefore use an automated algorithm to estimate source redshifts.
In doing this, the estimation selection rules are fixed and thus not tailored to each individual source. By automating the redshift estimation we increase the number of sources for which we can estimate redshifts, however in doing so we limit the efficiency of the estimation method by applying a 'one size fits all' approach.

Redshifts are estimated using a pseudo cross-correlation (PCC) method and stored. The PCC method works by first checking each spatial pixel in the sky, and determining whether it has a $120 \mu \mathrm{m}$ flux greater than our cutoff value, $S_{120 \mu m}>0.342 \mathrm{mJy}$. If true, the continuum emission is fitted with a fourth order polynomial, which is subtracted from the spectrum to leave an array containing only line emission and noise. The highest flux pixel in this array is considered to be a real emission line, and an array of possible redshifts is generated by assuming this line to be each of those in our set of template lines. The set of template lines is then placed at each of these possible redshifts and the redshift which gives the strongest correlation between our continuum subtracted spectra and the shifted template lines is taken as the best fit redshift. This process is then repeated on any unused possible emission lines in the spectrum to attempt to determine a secondary redshift. More details on the PCC method can be found in Raymond et al. (2009). Finally, estimated redshifts are compared to the 'truth' catalog in order to determine their accuracy.

\section{Results}

All results quantifying the redshift estimation in this section were determined by taking the average over the 100 SAFARI FoVs (with 10 different noise realizations for each SAFARI FoV: $1 \sigma=0.342 \mathrm{mJy}, 0$ mean) for both the burst mode and bright-end evolution models. When quoting results we give the number of accurately estimated $\left(\Delta z=\left|z_{\text {estimated }}-z_{\text {input }}\right|<0.1\right)$ redshifts as a percentage of the total number of sources brighter than the given cutoff flux. Conversely the number of inaccurate redshifts are given as a percentage of the total number of redshifts output by the algorithm.

For the bright-end and burst mode evolution models 37 and $53 \%$ of all primary sources with $S_{120 \mu m} \geq 0.342 \mathrm{mJy}$ are accurately estimated, with 14 and $9 \%$ of all redshifts output by the algorithm under the same constraints being inaccurate, respectively. For secondary sources (which exist for 6 and $8 \%$ of all spatial pixels) we accurately estimate redshifts for 37 and $29 \%$ of sources for bright-end and burst mode respectively, with 14 and $9 \%$ of all redshifts output by the algorithm under the same constraints being inaccurate, respectively. The distribution 120 of $\mu \mathrm{m}$ fluxes for sources with accurately estimated redshifts are shown in Figure 2.

The factor that impacts most on the efficiency of redshift estimation of the PCC method is emission line flux: it is easier to obtain redshifts for higher flux sources, as 

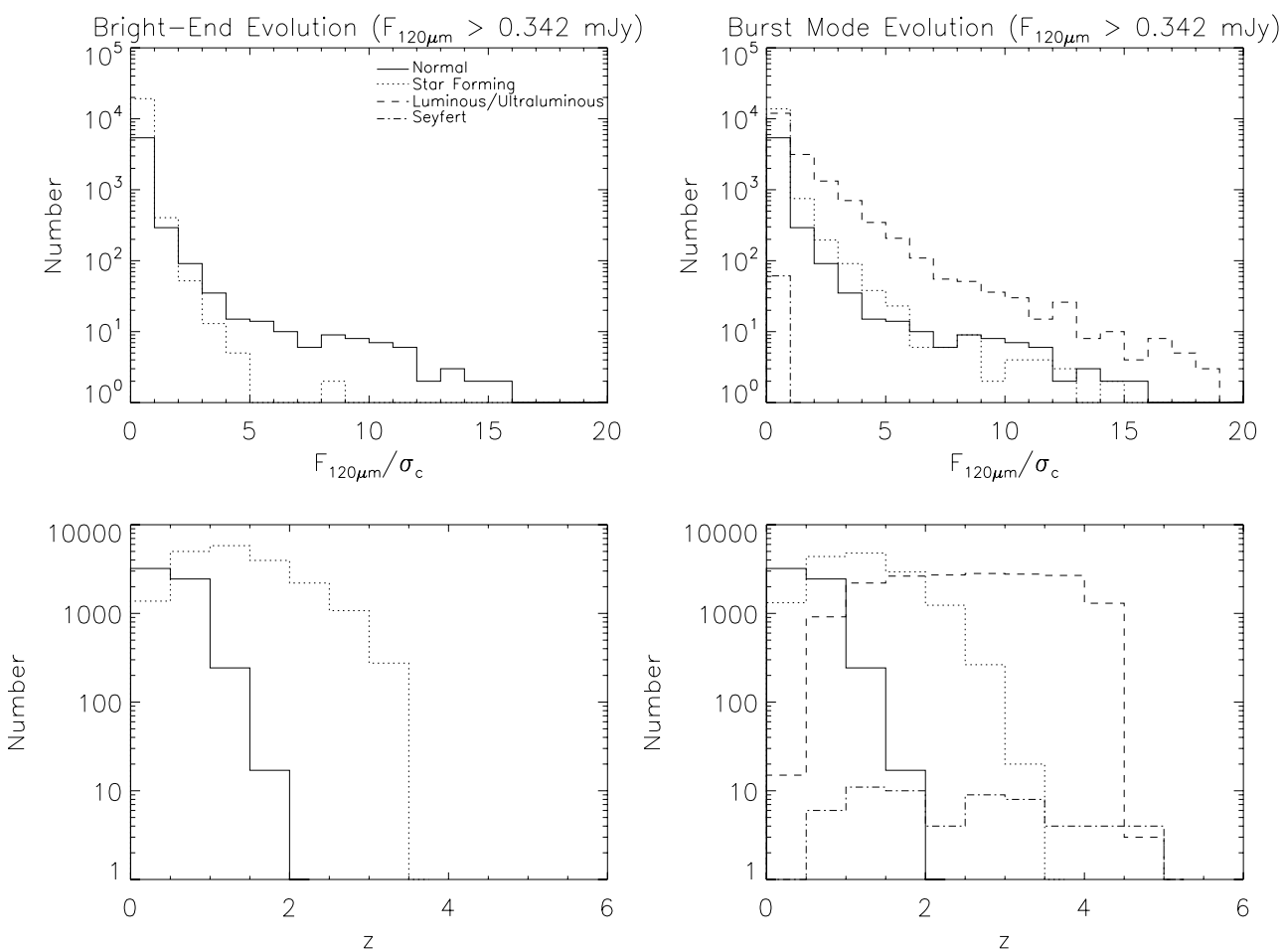

Figure 1. Plotted are the flux as a fraction of the $120 \mu \mathrm{m}$ confusion limit (top row) and redshift (bottom row) distributions of the sources that populate the bright-end and burst mode evolution data cubes respectively, for each spectral type. Each spectral type has the majority of sources at low fluxes, and beneath the confusion limit. The burst mode evolution model as opposed to the bright-end model however has more sources at higher fluxes. The bright-end evolution model contains only normal and star-forming spectral types whereas the burst mode includes, in addition, luminous/ultra luminous and Seyfert types. These more extreme spectral types in the burst mode model exist at higher redshifts than the other spectral types in either model. This means that the use of MIR emission lines is more important for the burst mode evolution model.

the emission lines are then more distinguishable against the instrumental noise. The burst mode evolution model has more sources at higher flux than the bright-end model, therefore we accurately estimate redshifts for a higher fraction of burst mode sources. The efficiency of the retrieval of accurate redshifts for secondary sources is also flux dependent. There are a number of additional factors that can hinder the accurate retrieval of redshifts for secondary sources. These include the flux ratio between the primary and secondary sources, as well as possible line degeneracies between the emission lines of the primary and secondary sources. Further work is required to quantify the these effects.

We can use a Kolmogorov-Smirnov test to establish whether we can use sets of estimated redshifts to differentiate between different evolutionary models. Thus, by comparing redshifts estimated from datacubes of different sizes, we can determine what area of sky needs to be surveyed by SAFARI in order to be able to reliably distinguish between different evolutionary models. We find than by using our PCC redshift estimation method we are able to reliably distinguish (probability that the sources from the two models are drawn from the same distribution, $P=0.01 \%$ ) between the bright-end and burst mode models with a sky survey area equal to 8 SAFARI FoVs, each of $10 \mathrm{hrs}$ integration time, taking a total total integration time of 80 hours.

\section{Conclusions}

We find that our PCC redshift determination method is capable of accurately estimating redshifts for sources that are more than an order of magnitude fainter than the traditional continuum confusion limit (at $120 \mu \mathrm{m}$ ). The efficacy of our method is higher for brighter sources. In this work we use the PCC method on model skies based on the parameters of the SAFARI instrument for SPICA, however this technique is also more generally applicable. The bright-end and burst mode evolution models include sources up to redshifts of $\mathrm{z} \sim 4$ and 5 respectively. At these redshifts we are still able to accurately estimate redshifts for sources using the PCC method. As yet, we have not tested to see the redshift at which the PCC method begins to fail.

The evolutionary models we have investigated in this work have the bulk of sources at fluxes fainter than the confusion limit, therefore by employing the PCC method we can greatly increase the number of galaxies that can be 

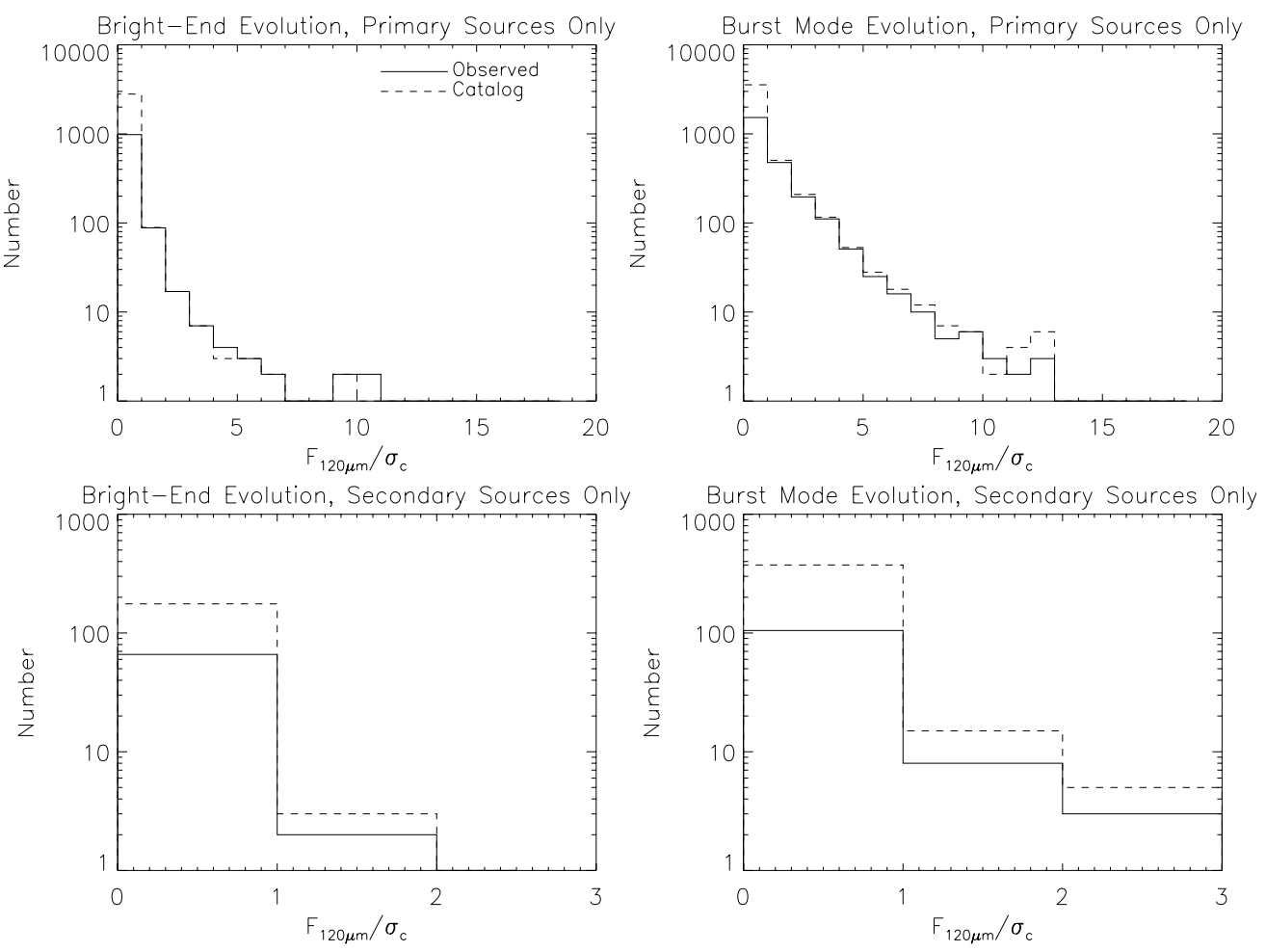

Figure 2. Plotted here are the the flux distributions for the input sources (dashed line) with $120 \mu \mathrm{m}$ flux $S_{120 \mu m}>0.342 \mathrm{mJy}$ and, those with redshifts accurately estimated by the PCC method (solid line). The top two panels are for primary sources and the bottom two are for secondary sources. The right two panels give results for bright-end evolution and the left two for burst mode. For both the evolution models the majority of sources lie at low fluxes. The bright-end has the highest concentration of low flux sources of the two models, and we accurately estimate redshifts for $37 \%$ of sources with $S_{120 \mu m}>0.342 \mathrm{mJy}$. The burst mode evolution model has more sources at higher fluxes, and as the most important factor in the efficiency of our PCC method is flux, has the higher accurate estimation rate of 53\%. Accurate redshifts are also estimated for 37 and $29 \%$ of secondary sources for bright-end and burst mode respectively.

uniquely identified in a single observation. This provides a larger statistical sample with which to compare observed source counts and redshift distributions with those presented in different evolutionary models.

Future work should include the investigation of the following; the use of the PAH features in identifying sources and determining their redshifts; the viability of taking into account sources with atypical line strengths; more realistic angular resolution modeling, where spatial resolution varies across the waveband; investigating the efficiency of the method when implemented on a wider range of evolutionary models. A detailed analysis of the effects of spectral type, initial luminosity and redshift parameters on our ability to accurately estimate redshifts from spectrally confused sources is also currently being undertaken.

\section{REFERENCES}

Clements, D. L., Isaak, K. G., Madden, S. C., Pearson, C. 2007, A\&A, 465, 125

Dole, H., et al. 2004, ApJS, 154, 93

Elbaz D., et al. 1999, A\&A, 351, L37
Jeong, W. -S., Pearson, C. P., Lee, H. M., Pak, S. J., Nakagawa, T. 2006, MNRAS, 369, 281

Le Borgne, D., Elbaz, D., Ocvirk, P., Pichon. C 2009, A\&A, 504, 727

Papovich, C., et al. 2004, ApJS, 154, 70

Pearson, C. P 2001, MNRAS, 325, 1511

Pearson, C. P. 2005, MNRAS, 358, 1417

Pearson, C. P., Jeong, W.-S., Lee, H. M., Nakagawa, T. 2007, Advances in Space Research, 40, 605

Pearson, C. P., Rowan-Robinson, M. 1996, MNRAS, 283, 174

Pilbratt, G. L. 2004, Presented at the Society of PhotoOptical Instrumentation Engineers (SPIE) Conference, 5481, 401

Raymond, G., Isaak, K. G., Clements, D. L., Rykala, A., Pearson, C. 2009, submitted

Scheuer, P. A. G. 1957, Proceedings of the Cambridge Philisophical Society, 53, 764 